\title{
Effectiveness of GeneXpert Technology in the Diagnosis of Smear-Negative Pulmonary Mycobacterium tuberculosis in HIV Positive Patients in Namibia
}

\author{
Ligamena N. Kakoma, Munyaradzi Mukesi*, Sylvester R. Moyo \\ Faculty of Health and Applied Sciences, Namibia University of Science and Technology, Windhoek, Namibia \\ Email: “mmukesi@nust.na
}

Received 1 July 2016; accepted 27 September 2016; published 30 September 2016

Copyright () 2016 by authors and Scientific Research Publishing Inc.

This work is licensed under the Creative Commons Attribution International License (CC BY).

http://creativecommons.org/licenses/by/4.0/

(c) (i) Open Access

\section{Abstract}

Tuberculosis (TB) remains a major public health problem accounting for more than 9.4 million incidents yearly of which most incidents are common in resource poor countries. The prevalence of Human Immunodeficiency Virus (HIV) in these countries is relatively high and TB/HIV co-infected patients often test negative for TB with direct microscopy, which poses diagnostic difficulties. The traditional diagnosis of HIV associated TB is complex, expensive, slow and technically demanding as it relies on conventional culture and drug susceptibility testing. The long delay required to obtain results has devastating consequences for patients who go undiagnosed or diagnosed too late. The purpose of the study was to determine the effectiveness of GeneXpert technology in the diagnosis of smear-negative pulmonary Mycobacterium tuberculosis in HIV positive patients. A retrospective cross sectional study was conducted on $\mathbf{4 0 0}$ patients suspected to have Mycobacterium tuberculosis and who were referred to the Namibia Institute of Pathology (NIP), Windhoek from November 2014 to May 2015. Patients with known HIV status who had their sputum tested by both the GeneXpert (Cepheid, California, United States of America) and direct microscopy (DM) for TB were included. Out of 400 sputum samples analysed for TB, $253(63.3 \%)$ cases were positive using GeneXpert whereas the DM was positive only for $133(33.3 \%)$ of the cases. Out of these 123 smear negative pulmonary tuberculosis (SN-PTB), 120 (98\%) sputum samples were from HIV positive patients while $3(2 \%)$ were from HIV negative patients. The sensitivity of the GeneXpert was calculated as $98 \%$ and the specificity was $52 \%$. The majority of the patients who contributed to SN-PTB were found to be co-infected with HIV. The GeneXpert's sensitivity was found to be high while the specificity was low. Even though this was the case, the GeneXpert as compared to DM could significantly reduce false negatives and the delay on treatment initiation can be significantly shortened, reducing premature death and ongoing transmission.

\footnotetext{
"Corresponding author.
} 


\section{Keywords}

\section{Tuberculosis, GeneXpert, Microscopy, HIV, Namibia}

\section{Introduction}

Tuberculosis (TB) is an airborne disease caused by the bacillus Mycobacterium tuberculosis. Tuberculosis usually infects the lungs but can also affect extra-pulmonary sites. Most infections with TB are caused by inhaling cough droplets or dust particles containing the tubercle bacilli which become lodged into the lung, forming an inflammatory lesion. Generally, people infected with M. tuberculosis will develop the disease, but the probability is much higher among HIV patients. Genus Mycobacteria infects a third of the world's population with $95 \%$ of those infected with M. tuberculosis species [1].

TB remains one of the world's deadliest communicable diseases. According to the World Health Organization (2014) approximately 9 million people developed TB in 2013. An estimated 1.1 million (13\%) of the 9 million people who developed TB in 2013 were HIV-positive. Namibia is the 4th leading country in TB incidence rate per 100,000 population with approximately 751 cases per 100,000 people. The data show that TB is a serious concern to the Namibian population given, the total population of 2.2 million [1].

If $\mathrm{TB}$ is to be eliminated as a global problem, earlier diagnosis, timely identification of rifampicin resistance as well as improved detection will be essential. Both diagnostic delays and treatment initiation can be significantly shortened, reducing premature deaths and on-going transmission [2].

Tuberculosis can remain dormant for years without showing symptoms or spreading to other people. The most common method for TB diagnosis worldwide is sputum smear microscopy or Direct Microscopy, which has limited sensitivity, especially among HIV-infected patients. Lack of prompt diagnosis and appropriate treatment of TB increases the risk of transmission, drug resistance and case fatality [3].

In 2007, the WHO decided to improve the diagnosis and management of smear-negative tuberculosis in HIV prevalent and resource constrained settings. The implementation required individuals with presumptive TB to be initially evaluated using two sputum microscopy examinations followed by clinical diagnosis such as chest $\mathrm{X}$-rays in smear-negative individuals [4].

Since that time, there has also been a lot of advancement in technology to assist with TB diagnosis such as the GeneXpert which uses Nucleic Acid Amplification Techniques (NAAT) to identify M. tuberculosis DNA and resistance to rifampicin. In December 2010, WHO endorsed the GeneXpert MTB/RIF for use in TB endemic countries and declared it a major milestone for global TB diagnosis [2]. However, during that period it was unclear how the assay performed as compared to the WHO 2007 algorithm in the diagnosis of smear negative pulmonary tuberculosis (SN-PTB) [4]. WHO's 2013 policy recommendations emphasised that after evaluating the GeneXpert MTB/RIF technology, it should be used rather than conventional microscopy and culture as the initial diagnostic test in adults suspected of multi drug resistant TB or HIV associated TB [5].

\section{Tuberculosis and HIV Co-Infection}

Tuberculosis is a potentially fatal contagious disease that can affect any part of the body but is mainly an infection of the lungs. Tuberculosis is caused by the tubercle bacillus, Mycobacterium tuberculosis (MTB). It is a contagious disease that spreads through air droplets when people with active disease cough, sneeze, sing or speak. The disease is of major public health importance in Namibia and one of the three most frequent causes of hospitalization and reason for attendance in the outpatient clinics [6]. If TB is left untreated, each person with active TB will infect on average of 10 and 15 people every year. This outlines the importance of correct diagnosis of TB to prevent further spreading of infection and help control the prevalence and incidence of TB infections in Namibia [7].

TB and HIV have been closely linked since the emergence of AIDS. The single most important factor contributing to the high prevalence and challenges in control of tuberculosis in developing countries is TB/HIV co-infection. Infection with HIV greatly increases the risk of developing tuberculosis and accelerates its progress. Progression to AIDS is also accelerated in those patients with TB/HIV co-infection. HIV greatly increases the progression of $\mathrm{TB}$, leading to more frequent extra-pulmonary involvement, atypical radiographic manifestations 
and paucibacillary disease which can impede timely diagnosis [8]. About $15.8 \%$ of the adult population in Namibia is HIV positive and $60 \%$ of TB patients are HIV positive indicating the serious burden of TB/HIV co-infection in Namibia. In Sub Sahara Africa, HIV is the most important cause of the increasing incidence of tuberculosis with up to $70 \%$ of those with tuberculosis being HIV positive [9].

While the GeneXpert technology has been widely received in Namibia, DSM is still widely used for the diagnosis of TB in HIV positive patients. The high HIV prevalence rate in Namibia makes effective TB diagnosis a priority in HIV positive patients to increase case detection and improve treatment outcomes. This study sought to evaluate the effectiveness of GeneXpert technology against conventional diagnostic techniques used in Namibia.

\section{Methods of TB Diagnosis}

Prompt and accurate TB diagnosis is the prerequisite for early and effective treatment thereby reducing tuberculosis burden. There is however a number of difficulties in achieving the WHO goal of eliminating TB. Sputum smear microscopy is inefficient due to its variable sensitivity particularly in patients with sputum smear-negative, extra pulmonary disease, and drug-resistant TB. Culture of MTB on Lowenstein-Jensen (LJ) medium is "the gold standard test", takes several weeks to produce results and hence causing delayed onset of treatment [10]. Recently, there have been significant advances in MTB diagnostic methods, including BACTEC, QuantiFERON-TB Gold, and nucleic acid amplification tests such as line probe assay and Gene-Xpert. A combination of factors including high costs, limited resources and the poor performance of various diagnostic tests make the diagnosis of TB difficult in developing countries [11].

\subsection{Smear Microscopy}

The most common way for diagnosing TB worldwide is through sputum smear microscopy/DM using the fluorescence microscope (Auramine) or the Ziehl-Neelsen method (gold standard). However this method is susceptible to human error and other factors beyond control that can result in false negatives. A number of factors that can cause false negatives include poor quality specimen or not obtaining the proper portion of the specimen during the smear preparation. Excessive decolourization during staining can also contribute to false negatives. There have also been cases where the issue has been associated with overheating of the slide during the fixing procedure, the slide being exposed to day light for too long in case of fluorescence microscopy and long interval between staining and reading of results [12].

The above mentioned factors can all be controlled with proper training and information given both to patient and the laboratory personnel involved with the testing. There is however one factor that is in most cases beyond human control and more to do with the pathophysiology of TB in HIV positive patients. This is false negative results in HIV positive patients because people with more advanced HIV tend to have lower levels of TB bacteria in their sputum. As a result, a larger proportion of people with HIV will test smear negative than in the general population [4].

The traditional way of diagnosing HIV associated TB and drug resistance is costly, time-consuming and technically demanding as it depends on conventional culture and drug susceptibility testing (DST). This process takes several days to obtain the results which have devastating consequences on patients who go undiagnosed or diagnosed too late, and it requires specialization and investment in laboratory infrastructure [2].

The impact of false negative in TB diagnosis can have far reaching consequences and is very detrimental to the global initiative as it may mean further spread of TB infections from untreated cases. Technology has however advanced and there are now automated methods to assist in the diagnosis such as the GeneXpert technology [2].

\subsection{Culture}

Several new techniques have been developed over years which are directed to detection of MTB directly from clinical specimen as well as for drug susceptibility testing. Lowenstein-Jensen medium is one of the non-selective medium used for recovery of Mycobacteria. It is therefore most commonly used in clinical diagnostic laboratories. It is however said to be less sensitive in the recovery of MTB from clinical specimens as compared to other culture media. The detection of growth is fairly prolonged up to 3 - 4 weeks. 
Several semi-automated and automated Mycobacteria detection methods are currently available, including radiometric BACTEC 460 system (BD Diagnostic System, Sparks MD) and the non-radiometric system, the MGIT 960 (BD Diagnostic System). The MGIT has an average detection time of positive samples of about 13 days as compared to the BACTEC 460 system which takes about 15 days and lastly, the culture method which takes about 27 days [13].

\subsection{GeneXpert MTB/RIF System}

The GeneXpert (Xpert MTB/RIF) is a cartridge based automated diagnostic test that can identify Mycobacterium tuberculosis (MTB) DNA and resistance to rifampicin (RIF) by nucleic acid amplification technique (NAAT). The Xpert MTB/RIF assay consists of two main components: 1) the Xpert MTB/RIF plastic cartridge, which contains liquid sample processing and PCR buffers and lyophilized real-time reagents 2) the GeneXpert instruments which controls intra-cartridge fluids and performs real-time PCR analysis. In December 2010 the World Health Organization (WHO) endorsed the Xpert MTB/RIF for use in TB endemic countries and declared it a major milestone for global TB diagnosis. The Xpert MTB/RIF provides sensitive detection of tuberculosis and rifampicin resistance directly from untreated sputum, in less than 2 hours with minimum hands-on time. This technology reduces "human error" and with the nucleic acid amplification technique (NAAT) it will reduce the number of false negatives diagnosed [14].

The GeneXpert system simplifies molecular testing by fully integrating and automating sample preparation, amplification and detection; required for real-time PCR testing. The Xpert MTB/RIF assay is based on heminested real-time PCR amplifying the rpoB gene target. Basically, target detection and characterization is performed in real time using a six-colour laser detection device. Molecular beacons using novel fluorophors and quenchers are used to detect hybridization to each of the five amplified target regions of the gene. Bacillus globigii, a spore-forming soil organism, is used as a full process control, acting as quality check for bacterial trapping, bacterial lysis, DNA extraction, amplification, and probe detection.

The assay was designed to be safe for use in microscopy laboratories where biological safety cabinets are usually not available. The sample treatment reagent is mycobactericidal, rapidly killing $M$. tuberculosis during the liquefaction process, and studies have confirmed that no aerosolation of Mycobacteria occur while running the device. The GeneXpert platform is FDA (Food and Drug Administration) approved and the assay is manufactured under ISO (International Organization for Standardization) 13485:2003 certification and approved by the regulatory authority in Europe.

Adoption of Xpert MTB/RIF does not eliminate the need for conventional TB microscopy, culture and DST capacity. Microscopy and culture remain necessary for monitoring of treatment, as it is unlikely that a test based on DNA detection would be suitable. Xpert MTB/RIF should therefore not be used for monitoring of treatment. In addition, conventional culture and DST are still required to detect resistance to anti-TB drugs other than rifampicin [15]. The study aimed to determine the effectiveness of GeneXpert technology in the diagnosis of smear-negative pulmonary Mycobacterium most significantly in HIV positive patients.

HIV positive patients have a decreased load of TB bacillus in their lungs because they usually form poor lung cavities when infected with TB, making it difficult to diagnose TB using direct microscopy alone as it may result in false negatives [4]. It was been reported that in $2011,430,000$ people were estimated to have died of TB and HIV co-infection. The risk of TB progressing from latent to active TB is estimated to be between 12 to 20 times greater in people living with HIV than among those without HIV infection. This also means that they may become infectious and pass TB on to other people at a faster rate than HIV negative patients [16].

\section{Research Design and Methodology}

\subsection{Research Design}

A retrospective-cross sectional study was conducted on patients suspected of Mycobacterium tuberculosis infection and who were referred to the Namibia Institute of Pathology (NIP) in Windhoek from November 2014 to May 2015. In the study TB was first routinely diagnosed by the use of microscopic examination of the sputum smear by means of auramine staining or Ziehl-Neelsen method. The final diagnosis was done using the GeneXpert. Patient data was including DM results, GeneXpert and HIV results was retrieved the laboratory information system. The sample size consisted of 400 sputum sample results of patients with known HIV status and their 
sputum samples tested by both the GeneXpert and direct microscopy for TB. Statistical analysis was performed using SPSS (Statistical Package of Social Sciences) software version 22. Chi-square was used to compare the two methods and ap-value equal to or less than 0.05 was considered to be statistically significant.

\subsection{Inclusion and Exclusion Criteria}

All patients with known HIV status and their sputum samples tested by both the GeneXpert and direct microscopy for TB at NIP from November 2014 to May 2015 were included in the study. All patients with unknown HIV status or patients who had been tested with only one method for PTB, being either the GeneXpert or the direct microscopy were excluded.

\subsection{Ethical Consideration}

Ethical clearance to conduct the study was obtained from, NIP, NUST and the Ministry of Health and Social Services (MoHSS) Research Committees. No individual consent was required as archived patient records were collected and no patient identification was used.

\section{Results}

The sample population of the study was 400 archived PTB patient records generated using the two methods; the GeneXpert and direct microscopy (DM) for TB diagnosis. Table 1 shows the comparison of the GeneXpert and DM in PTB detection.

Out of 400 sputum samples analysed for TB, the GeneXpert had more positive results $(63.3 \%)$ than DM (33.3\%). The difference between the two methods in PTB diagnosis was therefore statistically significant. Table 2 shows the comparison of the GeneXpert and DM in PTB detection according to HIV status.

Out of the SN-PTB (237), the majority of the sputum samples were from HIV positive patients (237) while only a few (30) were from HIV negative patients. The difference between the two methods in identifying TB in relation to HIV was statistically significant. Table 3 shows the diagnostic efficacy of the GeneXpert.

Table 1. The comparison of the GeneXpert and DM in PTB detection.

\begin{tabular}{llllll}
\hline & & & \multicolumn{2}{c}{ Direct Microscopy results } & Total \\
\cline { 4 - 5 } & & & Negative & Positive & \\
\hline \multirow{2}{*}{ GeneXpert results } & Negative & Frequency (\%) & $144(36.0 \%)$ & $3(0.8 \%)$ & $147(36.8 \%)$ \\
& Positive & Frequency (\%) & $123(30.8 \%)$ & $130(32.5 \%)$ & $253(63.3 \%)$ \\
& & Frequency (\%) & $267(66.8)$ & $133(33.3 \%)$ & $400(100 \%)$ \\
\hline
\end{tabular}

$\mathrm{p}$ value 0.0001

Table 2. The GeneXpert and DM comparison in PTB detection, adjusting for HIV.

\begin{tabular}{|c|c|c|c|c|c|c|}
\hline & \multirow{2}{*}{\multicolumn{2}{|c|}{ HIV status }} & & \multicolumn{2}{|c|}{ Direct Microscopy results } & \multirow{2}{*}{ Total } \\
\hline & & & & Negative & Positive & \\
\hline \multirow{4}{*}{ Negative } & & Negative & Frequency (\%) & $27(50.0 \%)$ & $0(0.0 \%)$ & $27(50 \%)$ \\
\hline & GeneXpert results & & & & & \\
\hline & & Positive & Frequency $(\%)$ & $3(5.6 \%)$ & $24(44.4)$ & $27(50.0 \%)$ \\
\hline & Total & & Frequency $(\%)$ & $30(55.6 \%)$ & $24(44.4 \%)$ & $54(100 \%)$ \\
\hline \multirow{3}{*}{ Positive } & & Negative & Frequency $(\%)$ & $117(33.8 \%)$ & $3(0.9 \%)$ & $120(34.7 \%)$ \\
\hline & GeneXpert results & Positive & Frequency $(\%)$ & $120(34.7 \%)$ & $106(30.6 \%)$ & $226(65.3 \%)$ \\
\hline & Total & & Frequency (\%) & $237(68.5 \%)$ & $109(31.5 \%)$ & $346(100 \%)$ \\
\hline
\end{tabular}

$\mathrm{p}$ value 0.001 . 
The Ziehl-Neelsen (DM) was used as the gold standard to determine the diagnostic specificity and sensitivity of the GeneXpert as culture was not routinely performed. The formulas below were used to calculate the GeneXpert sensitivity and specificity:

$$
\text { Sensitivity }=100(\text { True positive }) /(\text { True positive }+ \text { false negative })
$$

The diagnostic sensitivity was $98 \%$.

$$
\text { Sensitivity }=100(\text { True negative }) /(\text { True negative }+ \text { false positive })
$$

The diagnostic specificity was $54 \%$.

The sensitivity for the GeneXpert was $98 \%$ and the specificity was $54 \%$ respectively.

Table 4 is a comparison of the GeneXpert and DM in diagnosis of PTB cases in males and females.

The difference between the two methods (DM and GeneXpert) in detection of PTB according to gender was statistically significant. Figure 1 represents detection of PTB using GeneXpert and DM in the different age groups.

The age group with most SN-PTB was the 31 to 45 years (54\%). The difference between the two methods in PTB detection according to age group was statistically significant for age groups $>15$ years.

\section{Discussion}

The main objective of this study was to determine the effectiveness of the GeneXpert technology in the diagnosis of smear-negative pulmonary Mycobacterium tuberculosis in HIV positive patients using data from routine specimens for screening of PTB at NIP, Namibia from November 2014 to May 2015.

A total number of $253(63.3 \%)$ PTB cases were identified with the use of the GeneXpert whereas only 133 (33.3\%) of the TB cases were positive with DM as shown in Table 1. The difference in TB diagnosis between the two methods was statistically significant. The results are consistent with other studies and literature which states that DM has limited diagnostic benefits as it is susceptible to human error and other factors beyond control that can result in false negatives [3] [12]. In this study, factors related to false negatives may include poor quality specimens, not obtaining the proper portion of the specimen during smear preparation or the effect of

\begin{tabular}{|c|c|c|c|c|}
\hline & & \multicolumn{2}{|c|}{ GeneXpert results } & \multirow{2}{*}{ Frequency $(\%)$} \\
\hline & & Negative & Positive & \\
\hline \multirow{2}{*}{ DM as gold standard } & Patients without TB & $144(\mathrm{TN})$ & $123(\mathrm{FP})$ & $267(66.8 \%)$ \\
\hline & Patients with TB & $3(\mathrm{FN})$ & $130(\mathrm{TP})$ & $133(33.3 \%)$ \\
\hline \multicolumn{2}{|c|}{ Total } & $147(36.8 \%)$ & $253(63.3 \%)$ & $400(100 \%)$ \\
\hline
\end{tabular}

Table 3. Diagnostic efficacy of the GeneXpert.

$\mathrm{p}$ value $0.0001 ; \mathrm{TP}=$ True Positive $\mathrm{TN}=$ True Negative $; \mathrm{FN}=$ False Negative $; \mathrm{FP}=$ False Positive

\begin{tabular}{|c|c|c|c|c|c|c|}
\hline & \multirow{2}{*}{\multicolumn{2}{|c|}{ Gender }} & & \multicolumn{2}{|c|}{ Direct Microscopy results } & \multirow{2}{*}{ Total } \\
\hline & & & & Negative & Positive & \\
\hline \multirow{3}{*}{ Male } & \multirow{2}{*}{ GeneXpert results } & Negative & Frequency (\%) & $72(35.0 \%)$ & $1(0.5 \%)$ & $73(35.4 \%)$ \\
\hline & & Positive & Frequency (\%) & $68(33.0 \%)$ & $65(31.6 \%)$ & $133(64.6 \%)$ \\
\hline & \multicolumn{2}{|c|}{ Total } & Frequency (\%) & $140(68.0 \%)$ & $66(32.0 \%)$ & $206(100 \%)$ \\
\hline \multirow{3}{*}{ Female } & \multirow{2}{*}{ GeneXpert results } & Negative & Frequency $(\%)$ & $72(37.1 \%)$ & $2(1.0 \%)$ & $74(38.1 \%)$ \\
\hline & & Positive & Frequency $(\%)$ & $55(28.4 \%)$ & $65(33.5 \%)$ & $120(61.9 \%)$ \\
\hline & \multicolumn{2}{|c|}{ Total } & Count & $127(65.5 \%)$ & $67(34.5 \%)$ & $194(100 \%)$ \\
\hline
\end{tabular}

Table 4. Comparison of GeneXpert and DM in diagnosis of PTB according to gender of patients.

$\mathrm{p}$ value 0.0001 


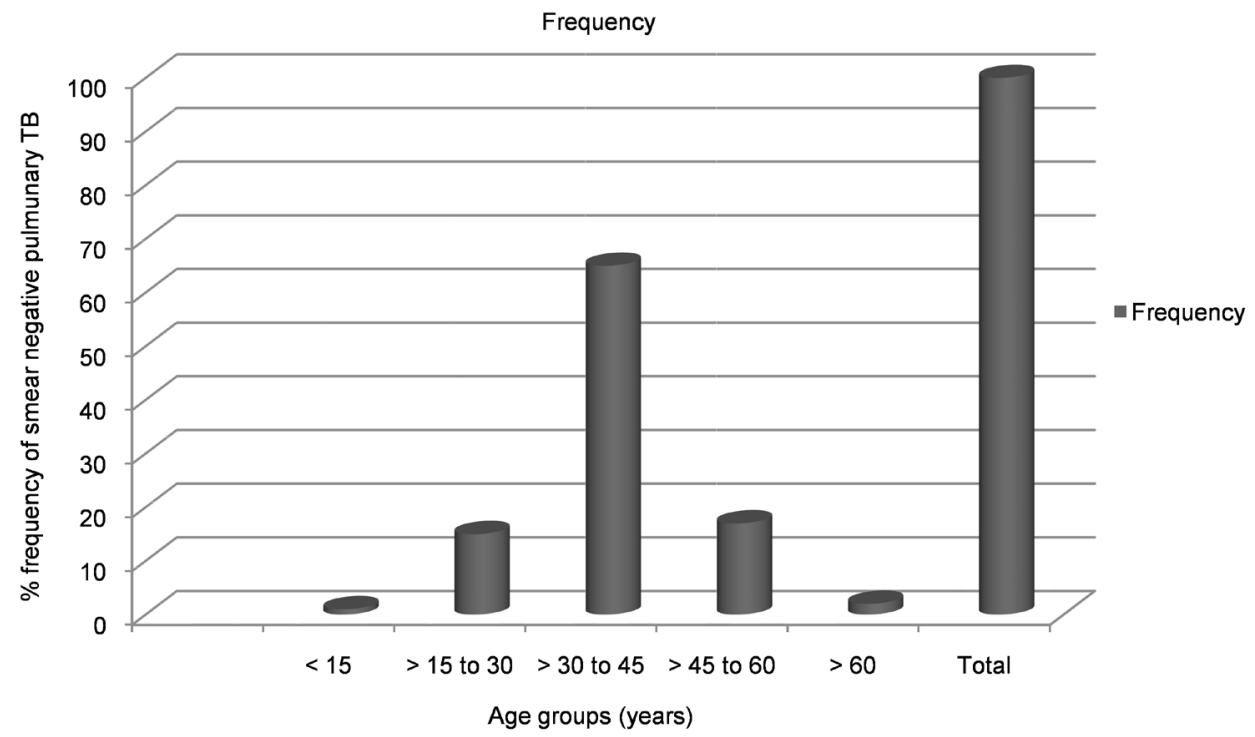

Figure 1. The GeneXpert and DM comparison in TB detection, adjusting for age groups ( $\mathrm{p}$ value $0.830)$.

PTB-HIV co-infection. It is disconcerting that most of these TB false negatives contribute to lack of prompt diagnosis and appropriate treatment and consequently increases the risk of transmission, drug resistance and case fatality. Our findings show that $3(0.8 \%)$ TB cases were detected with DM but the GeneXpert failed to detect them. GeneXpert targets only the MTB whereas DM is positive with all acid fast bacilli and thus not very specific.

Our findings indicate that SN-PTB was common in HIV positive patients when using DM as seen in Table 2. The findings of this current study agree with the results of other studies which noted that false negative results in HIV positive patients were a result of people with more advanced HIV having lower levels of TB bacteria in their sputum [4]. As a result, a larger proportion of people with HIV test smear negative than HIV negative people. Maximum benefit is therefore utilized by using the GeneXpert in not only targeting HIV/TB co-infected patients but also paediatric patients, drug-resistant TB as well as extra-pulmonary TB. Consequently, diagnostic delay and treatment initiation can be significantly shortened, reducing premature death and on-going transmission [15].

Table 3 shows that the GeneXpert had a sensitivity of $98 \%$ and the specificity of $54 \%$, respectively when compared to the Ziehl-Neelson method. Other studies have reported high specificity and sensitivity of the GeneXpert method as in comparison to the results of this current study. A study conducted by WHO (2011) indicated the GeneXpert sensitivity of $80 \%$ and the specificity of more than $80 \%$ in patients with smear negative PTB [15]. When the GeneXpert was compared to the TB culture gold standard the sensitivity was $67 \%$ while the specificity was $98 \%$ [14]. In a similar study the GeneXpert sensitivity was $95 \%$ and the specificity $33 \%$ [4]. The results of this current study could be different from other studies as we used the Ziehl-Neelsen method as the gold standard as compared to other studies that used culture.

In HIV/PTB co-infection in SN-PTB, $120(34.7 \%)$ patients were HIV positive while only $3(0.9 \%)$ patients were HIV negative as shown in Table 2. Comparing our results with other studies, our findings indicated a big difference. DM positive and GeneXpert positive results on the other hand show that $3(0.9 \%)$ were HIV positive. The observed difference between the two methods among HIV positive patients and HIV negative patients in this study and in other similar studies is due to the impact of HIV in causing false positive results with DM than with the GeneXpert [15].

Our findings indicate that SN-PTB was common in HIV positive patients when using DM as seen in Table 2. The findings of this study agree with the results of other studies which showed that false negative results in HIV positive patients were a result of people with more advanced HIV having lower levels of TB bacteria in their sputum [4]. As a result, a larger proportion of people with HIV test smear negative than HIV negative people. Maximum benefit is therefore utilized by using the GeneXpert in not only targeting HIV/TB co-infected patients 
but also paediatric patients, drug-resistant TB as well as extra-pulmonary TB. Consequently, diagnostic delay and treatment initiation can be significantly shortened, reducing premature death and on-going transmission [15].

This study found that men had more SNMTB $68(33.0 \%)$ than women 55(28.4\%) as shown in Table 4. This is in agreement with other studies including a study by WHO [17] [18]. These results however are only based on global statistics in developed countries. Currently, there is no conclusive evidence of gender-based difference in the occurrence of TB and HIV-co-infection in developing countries.

As shown in Figure 1, the most affected age category with SN-PTB was $>31$ to $\leq 45$ years $(80 \%)$, followed by $>45$ to $\leq 60$ years (21\%). The economically productive age groups are primarily affected and thus impacting the Namibian society in terms of loss of economic productivity due to absenteeism, loss of potential tax revenue, loss of trained human resources and ever-rising health care costs. This is consistent with findings of other studies [6] [18]. The age preponderance to SN-PTB could also be due to the fact that these groups are sexually active, therefore encountering sexual partners in whom both TB and HIV are both prevalent [18].

\section{Conclusion}

Most of the people with SN-PTB were co-infected with HIV. Furthermore, the GeneXpert's sensitivity was found to be high while the specificity was low. Even though this is the case, the GeneXpert as compared to DM can significantly reduce false negatives and the delay on treatment initiation can be significantly shortened, reducing premature death and ongoing transmission. Further studies are needed to define the impeding factors and to identify a simple and efficient way to enhance the specificity of the GeneXpert for detection of Mycobacterium tuberculosis in HIV/TB co-infected patients.

\section{Acknowledgements}

The authors would like to acknowledge Dr. Sidney Chikukwa, Namibia Institute of Pathology and the Ministry of Health and Social Services, Namibia.

\section{References}

[1] WHO Global Tuberculosis Report 2014. http://www.who.int/tb/publications/global_report/gtbr14_main_text.pdf

[2] Weyer, K., Mirzayev, F., Migliori, G.B., Van Gemert, W., D’Ambrosio, L., Zignol, M. and Raviglione, M. (2013) Rapid Molecular TB Diagnosis: Evidence, Policy Making and Global Implementation of Xpert MTB/RIF. European Respiratory Journal, 42, 252-271. http://dx.doi.org/10.1183/09031936.00157212

[3] Boehme, C.C., Nabeta, P., Hillemann, D., Nicol, M.P., Shenai, S., Krapp, F. and Perkins, M.D. (2010) Rapid Molecular Detection of and Rifampicin Resistance. New England Journal of Medicine, 363, 1005-1015. http://dx.doi.org/10.1056/NEJMoa0907847

[4] Walusimbi, S., Bwanga, F., De Costa, A., Haile, M., Joloba, M. and Hoffner, S. (2013) Meta-Analysis to Compare the Accuracy of GeneXpert, MODS and the WHO 2007 Algorithm for Diagnosis of Smear-Negative Pulmonary. BMC Infectious Diseases, 13, 507. http://dx.doi.org/10.1186/1471-2334-13-507

[5] WHO Xpert MTB/RIF Implementation Manual 2014. http://www.who.int/iris/bitstream/10665/112469/1/9789241506700_eng.pdf

[6] MoHSS (2010) National and Leprosy Control Programme 2010-2015 Annual Report. Directorate of Special Programmes, Windhoek.

[7] Menzies, N.A., Cohen, T., Lin, H.H., Murray, M. and Salomon, J.A. (2012) Population Health Impact and Cost-Effectiveness of Diagnosis with Xpert MTB/RIF: A Dynamic Simulation and Economic Evaluation. PLoS Med, 9, e1001347. http://dx.doi.org/10.1371/journal.pmed.1001347

[8] Luetmeyer, A., Gooze, L. and Daley, C.L. (2013) Comprehensive, up to Date Information on HIV/AIDS Treatment, Prevention and Policy from the University of California and San Francisco. http://hivinsite.ucsf.edu

[9] Index Mundi (2014) Namibia Demographic Profile 2014. http://www.indexmundi.com/namibia

[10] Arora, D., Jindal, N., Bansal, R. and Arora, S. (2015) Rapid Detection of Mycobacterium in Sputum Samples by Cepheid Xpert Assay: A Clinical Study. Journal of Clinical and Diagnostic Research, 9, DC03-DC05. http://dx.doi.org/10.7860/jedr/2015/11352.5935

[11] Venturini, E., Turkova, A., Chiappini, E., Galli, L., de Martino, M. and Thorne, C. (2014) Tuberculosis and HIV 
Co-Infection in Children. BMC Infectious Diseases, 14, S1-S5. http://dx.doi.org/10.1186/1471-2334-14-S1-S5

[12] Cheesbrough, M. (2006) District Laboratory Practice in Tropical Countries: Part Two. 2nd Edition, Cambridge University Press, Cambridge. http://dx.doi.org/10.1017/cbo9780511543470

[13] Winn, W.C. (2006) Koneman's Color Atlas and Textbook of Diagnostic Microbiology. E. W. Koneman, 6th Edition, Lippincott Williams \& Wilkins, Philadelphia.

[14] Ioannidis, P., Papaventsis, D., Karabela, S., Nikolaou, S., Panagi, M., Raftopoulou, E. and Kanavaki, S. (2011) Cepheid GeneXpert MTB/RIF Assay for Mycobacterium Detection and Rifampin Resistance Identification in Patients with Substantial Clinical Indications of and Smear-Negative Microscopy Results. Journal of Clinical Microbiology, 49, 3068-3070. http://dx.doi.org/10.1128/JCM.00718-11

[15] WHO (2011) Automated Real-Time Nucleic Acid Amplification Technology for Rapid and Simultaneous Detection of and Rifampicin Resistance: Xpert MTB/RIF System.

[16] World Health Organisation (2014) http://www.who.int/mediacentre/factsheets/who104/en/print.html

[17] World Health Organization (2013) Automated Real-Time Nucleic Acid Amplification Technology for Rapid and Simultaneous Detection of and Rifampicin Resistance: Xpert MTB.

[18] Agbor, A.A., Bigna, J.J.R., Plottel, C.S., Billong, S.C., Tejiokem, M.C., Ekali, G.L. and Koulla-Shiro, S. (2015) Characteristics of Patients Co-Infected with HIV at the Time of Inpatient Treatment Initiation in Yaoundé, Cameroon: A Tertiary Care Hospital-Based Cross-Sectional Study. Archives of Public Health, 73, 24. http://dx.doi.org/10.1186/s13690-015-0075-y

\section{Submit or recommend next manuscript to SCIRP and we will provide best service for you:}

Accepting pre-submission inquiries through Email, Facebook, LinkedIn, Twitter, etc.

A wide selection of journals (inclusive of 9 subjects, more than 200 journals)

Providing 24-hour high-quality service

User-friendly online submission system

Fair and swift peer-review system

Efficient typesetting and proofreading procedure

Display of the result of downloads and visits, as well as the number of cited articles

Maximum dissemination of your research work

Submit your manuscript at: http://papersubmission.scirp.org/

Or contact ojmm@scirp.org 